\title{
Crystal structure information in drug discovery and development: current perspectives and new possibilities from the Cambridge Crystallographic Data Centre
}

\author{
"Crystal structure knowledge, at both the molecular and supramolecular levels, is embedded in procedures that aid rational \\ drug discovery and development on a day-to-day basis."
}

In its quest for new active pharmaceutical ingredients (APIs), the pharmaceutical industry depends heavily on precise information about molecular structure (particularly molecular shape) and intermolecular interactions. Once discovered, each new API must then be developed into a marketable formulation. This requires knowledge of the properties of pharmaceutical materials, particularly in solid form. While medicinal chemists, modelers and development scientists have a plethora of tools available to them, knowledge of experimental structure in its broadest sense is an absolute prerequisite for both discovery and development.

Crystal-structure analysis is the method of choice for structure determination across the complete chemical spectrum, from inorganics through organics and metal organics to proteins and viruses. The results provide precise experimental data on molecular geometry, conformation and higher order features in macromolecules. Additionally, because crystal structures comprise essentially infinite symmetric molecular arrays, they are unique in providing direct experimental observations of intermolecular interactions, yielding the geometries and motifs formed by hydrogen bonds and other nonbonded interactions, which are fundamental to our understanding of both protein-ligand interactions and the properties of pharmaceutical materials in solid form. Crystals are truly "windows on the world of atoms" [1] and the results of nearly 700,000 crystal structure determinations are currently available in a number of fully retrospective and comprehensive databases [2-6]. This overall total increases by more than 45,000 new structures per year.

The most relevant of these databases to pharmaceutical discovery and development are the Protein Data Bank (PDB) [5] and the Cambridge Structural Database of organic and metal organics (CSD) [6]. In February 2010, the PDB contained 63,559 protein structures and it increases in size by more than 7000 structures per year. The CSD has recently recorded its 500,000th structural entry and increases in size by more than 35,000 structures per year. This profile summarizes the structural knowledge relevant to drug discovery and development that can be obtained using the CSD and its associated software.

\section{The Cambridge Structural Database System}

Compilation of the CSD began in 1965 and the information content of a structural entry is fully described elsewhere [6]. In summary, the CSD records data from single-crystal x-ray and neutron diffraction studies of C-containing compounds: organics and metal organics. Each entry records the primary numerical results of the analysis: 3D atomic coordinates, cell dimensions and space group, together with a formal $2 \mathrm{D}$ chemical diagram and searchable chemical connection table, full bibliographic reference, including the digital object identifier, chemical name, formula and other information that may be present in the published report. The CSD also contains over 6000 structures deposited directly by crystallographers.

The complete CSD System also includes the software applications ConQuest, Mercury and Vista. ConQuest permits searches of all CSD information fields, most importantly for chemical substructures and intermolecular interactions defined using both $2 \mathrm{D}$ connectivity and $3 \mathrm{D}$ geometric constraints [7]. Apart from locating relevant crystal structures, ConQuest also outputs user-defined geometrical descriptors for the structure or substructure of interest. Mercury is a 3D structure visualizer with facilities to view crystal

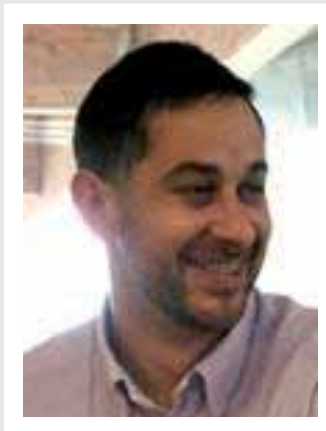

Colin R Groom

Author for correspondence: Cambridge Crystallographic Data Centre (CCDC), 12 Union Road, Cambridge, CB2 IEZ, UK Tel.: +44 I223336020 Fax: +44 1223336033 E-mail: groom@ccdc.cam.ac.uk

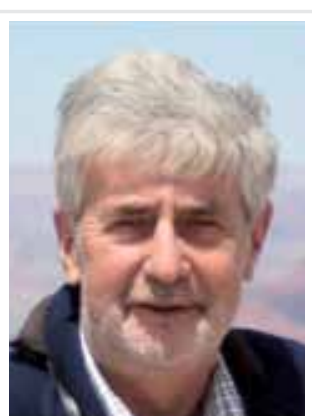

Frank H Allen

Cambridge Crystallographic Data Centre (CCDC), 12 Union Road, Cambridge, CB2 IEZ, UK 


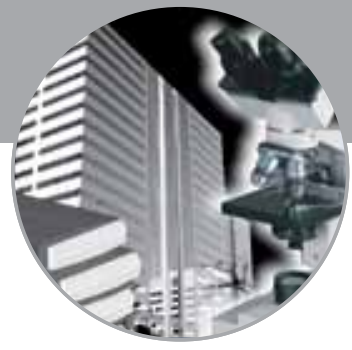

packing, build $\mathrm{H}$-bonded motifs and display intermolecular interaction patterns [8]. Work to extend Mercury to aid pharmaceutical materials development is ongoing. Vista is an application that permits geometrical data retrieved from the CSD to be analyzed statistically and displayed as histograms and scattergrams. Two knowledge bases, MOGUL, a knowledge base of intramolecular geometry [9], and IsoStar, a knowledge base of intermolecular interactions [10], are also part of the distributed system. They provide rapid access to CSD information that is crucial in drug discovery and development, as indicated below. Recently, the CSD has been made internet accessible through the WebCSD application [11], which also incorporates $2 \mathrm{D}$ chemical similarity searches of the CSD. An intranet application for inhouse networks is also available.

\section{Conformational preferences}

The fundamental importance to drug discovery of the conformational analysis of small molecules has been extensively reviewed by Stahl and co-workers [12]. They note that a knowledge of the energetically accessible conformations available to both molecules and their constituent substructures can be obtained using computational methods or by using experimental data provided by crystal structures. Molecular conformations can be obtained directly from the CSD or from PDB data on protein-bound ligands, but the real value of atomic-resolution CSD information arises from its massive chemical diversity; it provides large numbers of observations of different organic substructures in a wide range of crystal-packing environments.

Additionally, the CSD has excellent coverage of what these authors term 'pharmaceutical chemical space'. Thus, the database contains the structures of almost 60,000 small molecules that obey a modification of the commonly used 'rule of five' [13,14], originally developed as a guide to the likelihood of achieving oral bioavailability in a drug, but often used as a surrogate to describe drug-likeness. Of course many more molecules in the CSD contain drug-like fragments, which provide valuable information, despite being part of a non drug-like molecule. By contrast, PDB ligand data are normally determined at lower resolution and lack the chemical diversity of the CSD. Furthermore, it is noted that small-molecule crystal structure conformations generally correlate well with protein-bound conformations, so that torsion angle libraries derived from the CSD have added value in modeling novel ligands in pharmaceutical applications [12].
However, the question of whether crystal structure conformations are affected by packing effects, such as conformational adjustments that facilitate highly favorable nonbonded interactions, is frequently raised. Another recent review provides a summary of conformational analysis methodologies that have been applied to CSD data and specifically addresses evidence concerning packing effects [15]. Both reviews conclude that the very few well-known examples of higher-energy conformations observed in crystal structures are the exception rather than the rule $[12,15]$. For example, comparisons of CSD torsional distributions with ab initio energy curves and extensive studies of the conformations adopted by the many CSD molecules that exist in more than one crystal environment both show that:

- Torsions associated with a calculated strain energy of at least $5 \mathrm{~kJ} \mathrm{~mol}^{-1}$ are observed in less than $3 \%$ of crystal structure conformations;

- Higher energy conformations may be under-represented in crystal structures;

- Crystal structure conformations are good guides to the conformational preferences of free molecules, especially where reasonably large numbers of experimental results are available.

With over half a million structural entries, the CSD provides a plethora of conformational data. This has been collected in the MOGUL knowledge base of intramolecular geometry [9], which contains more than 20 million bond lengths, valence angles and torsion angles organized into more than 1.8 million parameter distributions that can be searched using a chemical hierarchy. Structures of interest can either be drawn into the MOGUL interface or 3D models can be imported in a variety of common formats. Clicking on two, three or four atoms in the model will generate a distribution of bond lengths, valence angles or torsions for the appropriate chemical environment. The example in Figure I shows the MOGUL distribution of $\mathrm{C}_{\mathrm{ar}}-\mathrm{C}_{\mathrm{ar}}-\mathrm{S}-\mathrm{C}$ torsions in arylsulfones, with the torsion from the input model indicated and with full hyperlinking of the parameter distribution back to the appropriate CSD entries. Options also exist to generate torsional distributions for all freely rotatable bonds in the model molecule and to check the geometry of an input model, such as a protein-ligand docking pose, against the experimental 'reality' of crystal structure geometries. 


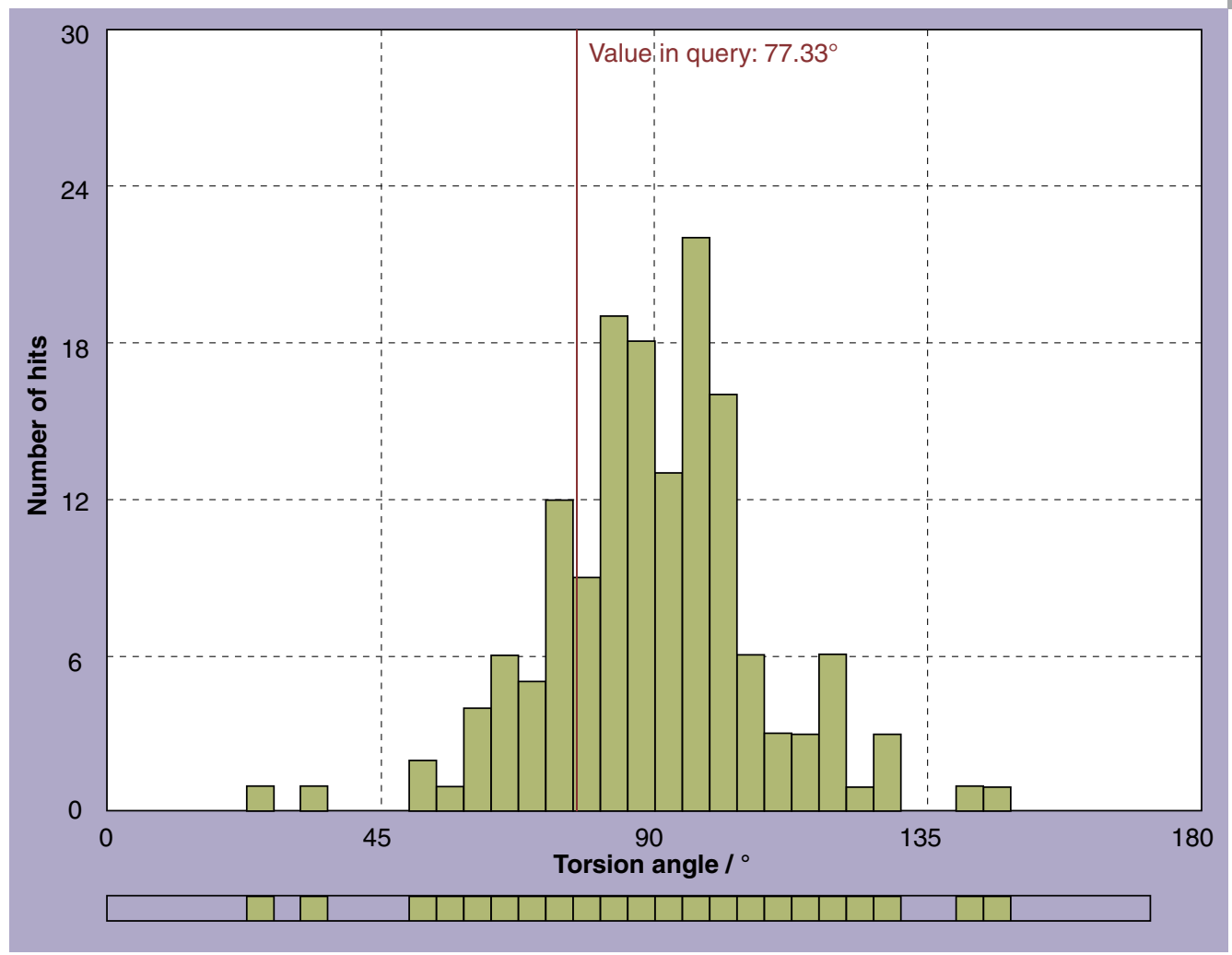

Figure 1. Distribution of $\mathrm{C}_{\mathrm{ar}}-\mathrm{C}_{\mathrm{ar}}-\mathrm{S}-\mathrm{C}$ torsion angles in arylsulfones from the Cambridge Crystallographic Data Centre's MOGUL knowledge base.

Torsional information from the CSD has a long history of use in drug discovery, as discussed by Taylor [16], contributing to early $2 \mathrm{D} \rightarrow 3 \mathrm{D}$ structure converters that generate a single $3 \mathrm{D}$ conformer. The later program MIMUMBA [17] is a knowledge-based program for generating all low-energy conformers, in which the knowledge resides in a torsion library derived from the CSD for 216 common molecular fragments. This technology for conformer generation was further improved by workers at Merck in their program known as enumerate torsions (ET) [18], which used a library of some 800 flexible torsions and took better account of correlations between the torsion angles of adjacent rotatable bonds. Applications of CSD-derived torsional libraries within protein-ligand docking software are noted below.

\section{Intermolecular interactions}

Crystal structure analyses are central to our understanding of hydrogen bonds and other nonbonded interactions not mediated by hydrogen. No other technique provides such precise metrical and spatial descriptions of intermolecular interactions and, since its inception, the CSD has been a key data source, contributing information to many hundreds of research papers and several monographs [19]. CSD analysis of H-bonds has quantified, for example [20], lone pair directionality at acceptor atoms, the characteristics of resonance-assisted and -induced $\mathrm{H}$-bonds, the existence of $\mathrm{C}-\mathrm{H} \cdot \bullet \times \mathrm{X}$ bonds, $\mathrm{H}$-bond competition effects and the formation of preferred H-bond motifs that act as synthons in crystal engineering applications. The CSD also provides evidence for strong and sometimes structure-directing dipole-dipole interactions, such as $\mathrm{CO} \cdot \bullet \mathrm{CO}$ and $\mathrm{CN} \cdot \cdots \mathrm{CN}$. Two recent articles by Stahl and co-workers highlight the importance to medicinal chemists of experimental knowledge of intermolecular interactions and intramolecular hydrogen bonding [21,22].

However, while crystal-structure information defines precise geometries, it can only provide qualitative information about interaction energies based on geometrical differences. Hence, in recent years, CSD analysis has been used extensively in conjunction with ab initio methods to provide high-quality interaction energies [15]. The CSD is used to identify preferred interaction areas on the potential energy hypersurface and the relatively expensive ab initio calculations then examine these areas in detail. These studies further extend our knowledge and improve our ability to predict which interactions are 
most likely to be crucial in, for example, protein-ligand binding, crystal engineering and polymorph formation.

The CSD knowledge base IsoStar provides visual and numerical information about a wide variety of nonbonded interactions [10]. IsoStar provides 2D scatterplots of the distribution of a contact group (e.g., a H-bond donor or other functional group) around a central group, exemplified by the 'native' and contoured plots of N-H donors around an amide central group, as seen in Figure 2. IsoStar data are derived from the CSD (>20,000 scatterplots), from protein-ligand complexes in the PDB with a resolution better than $2 \AA$ (>5500 scatterplots) and theoretical energy minima for more than $1500 \mathrm{key}$ interactions. IsoStar also provides geometrical statistics and all scatterplot points are hyperlinked to the crystal structures in which they were observed. IsoStar has obvious general applications in both drug discovery and drug development.

\section{Protein-ligand interactions \& protein-ligand docking}

The environment surrounding a small molecule in a crystal lattice is not unlike that found in the ligand-binding cavity of a protein. Thus, the structure-based information on preferred interaction geometries contained in IsoStar is used as a knowledge base by the SuperStar program [23] to predict interaction hot spots in a binding cavity (the gold areas in Figure 3) for the functional groups of a putative ligand. These interaction maps can then inform discovery scientists about where functional groups might be placed in a molecule to increase the interaction energy. The gold areas in Figure 3 are generated by SuperStar for a carbonyl $(\mathrm{C}=\mathrm{O})$ probe in the binding cavity of tyrosine kinase and are overlayed with the structure of an oxindole-based inhibitor (SU4502) determined by crystal structure analysis of the kinase-inhibitor complex [24]. The binding of the two inhibitor $\mathrm{C}=\mathrm{O}$ groups, from its oxindole and carboxylic acid functions, are seen to be well predicted by SuperStar, as hydrogen-bonded interactions with the kinase ALA564 and ASN568 residues, respectively. However, SuperStar also suggests other areas of the cavity that would be attractive to $\mathrm{C}=\mathrm{O}$ and suggests that inhibitor binding might be improved if its chemical structure was suitably modified. SuperStar can also be used to generate a pharmacophoric pattern from a collection of 'hot spots' generated for different functional groups, further assisting in the modification of existing candidates, or in the identification of new novel ligands for binding in a specific protein active site.

Protein-ligand docking is now a well-established computational methodology for the virtual screening of proposed new lead compounds and small-molecule crystal structure information plays an important role in the leading software offerings [16]. Both GOLD [25] and FlexX [26] make use of torsional distributions from the CSD, either to focus the conformational search into favored regions of conformational space (GOLD) or to build up the ligand conformation from rigid fragments (FlexX). CSD information on hydrogen bonding also
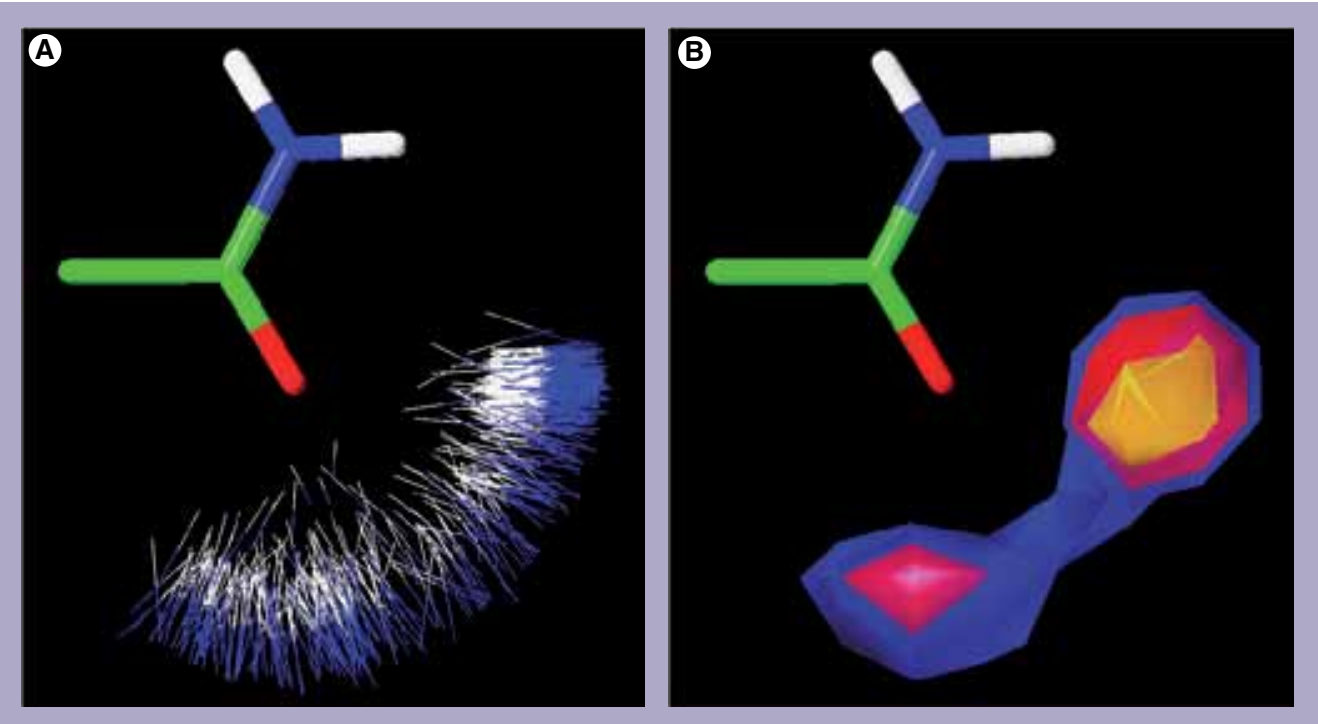

Figure 2. IsoStar scatterplots of an $\mathrm{N}-\mathrm{H}$ contact group around an amide central group: (A) standard plot and (B) contoured plot. 

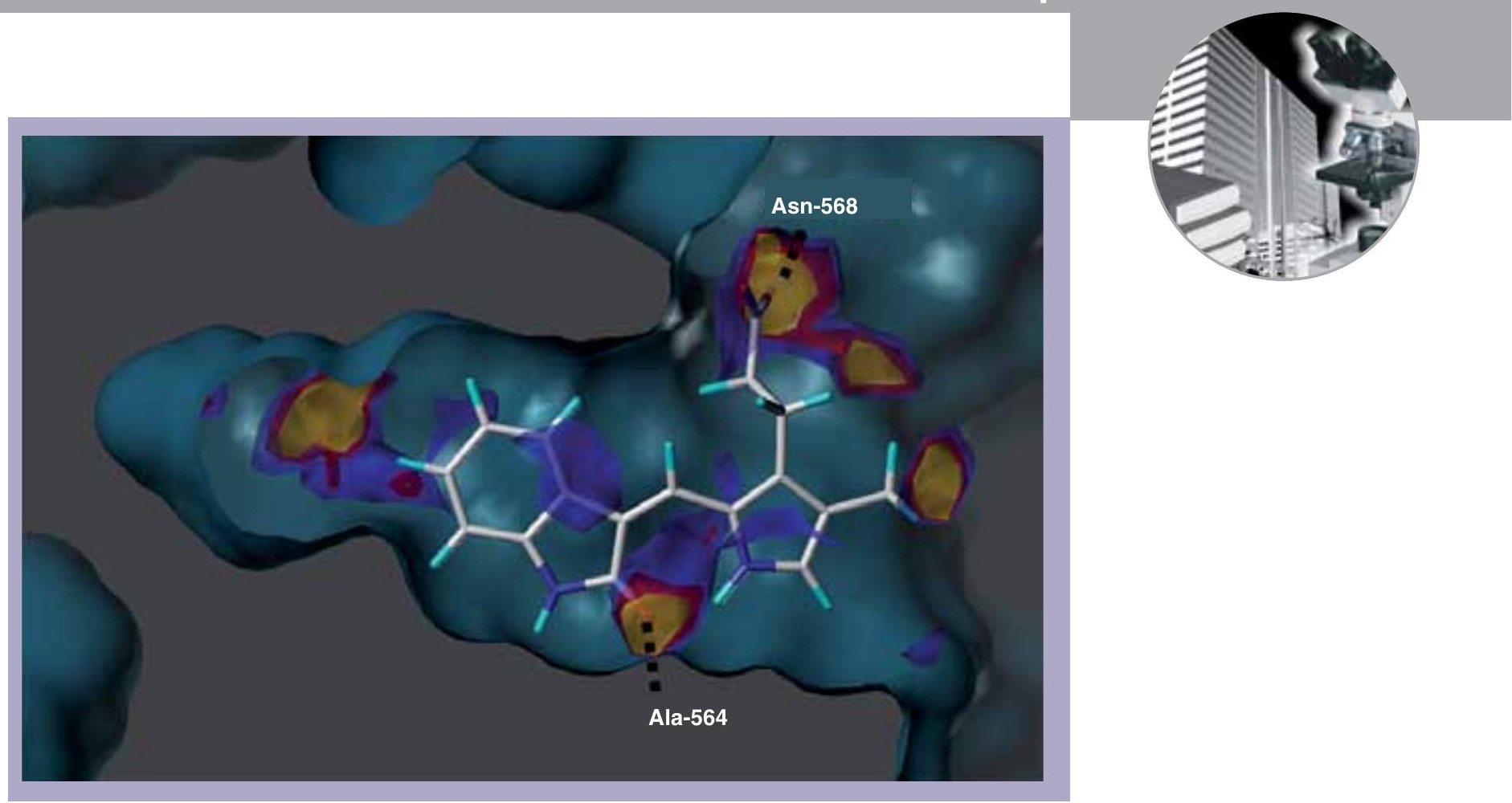

Figure 3. Interaction 'hot spots' for a $\mathrm{C}=\mathrm{O}$ group within the binding site of tyrosine kinase [21] (shown in gold), with the actual position of an oxindole inhibitor

superimposed. The correspondence of the two inhibitor $\mathrm{C}=\mathrm{O}$ groups with two of the SuperStar hot spot predictions is clearly observed.

assists decision making in the genetic algorithm employed in GOLD. A key use of interaction data derived from the CSD is in the generation of protein-ligand scoring functions, for example, DrugScore CSD [27]. While it is still difficult to use these functions to predict binding energy, they are nevertheless of great value in identifying ligands that have the potential to bind to a particular protein and can, to some degree, rank such ligands. In many cases, these functions are also able to rank different poses of the same ligand in a protein-binding site. Reproduction of the binding mode observed between a particular ligand and a protein can be fairly readily achieved, but many difficulties present themselves in so called cross-docking problems. Finally, a vital component of identifying a likely ligand-binding mode is the assessment of the conformation of the bound ligand. This can be done using theoretical calculations (often based on CSD data), but is perhaps most effectively performed by reference to experimental structures, using the MOGUL system, as described earlier.

\section{Pharmaceutical materials}

The rational design of solid state forms of APIs is a topic of major current interest. In 2002, the Pfizer Institute for Pharmaceutical Materials Science (PIPMS) [101] was established at the
University of Cambridge and the Cambridge Crystallographic Data Centre (CCDC). Major research topics have included, inter alia, salt selection, solvation, counter-ion motifs, cocrystal formation and its prediction, polymorphism and polymorph screening, ab initio crystal structure and property prediction and structure determination using modern powder diffraction [28]. Much of this work is aimed at a deeper understanding of solid forms, particularly in the crystalline state and has led to some significant advances in enabling technology, involving novel search and analysis of the CSD and further applications of derived structural knowledge, as exemplified in a recent review [29]. Thus, the Mercury program [8] has been enhanced to search for the following:

- Interaction motifs involving a specified set of functional groups;

- Packing features observed in an existing structure - what other structure types exhibit these features?

- Analysis of crystal-packing similarity to locate groups of similar extended structures among families of polymorphs.

Software development and basic research go hand in hand at the CCDC and current research includes the derivation of $\mathrm{H}$-bond propensity 


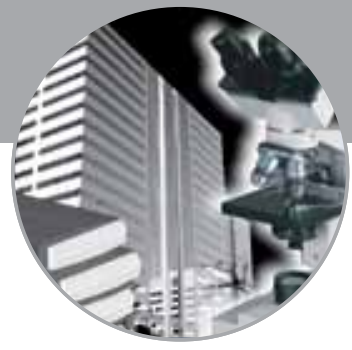

data from the CSD and its application in a statistical methodology for informatics-based polymorph screening based only on the $2 \mathrm{D}$ chemical diagram of an API. A recent application to ritonavir is revealing [30]. This anti-HIV agent was first successfully formulated in 1997 by Abbott Laboratories using a crystalline product (now known as Form I). However, in 1998, a second, more stable, but far less soluble, Form II appeared in production leading to a very costly product withdrawal until the problem could be resolved. H-bond propensities $(\pi)$ were derived from the CSD for the six $\mathrm{H}$-bonding functional groups of ritonavir and analysis showed that the two $\mathrm{H}$-bonds with the lowest $\pi$-values were involved in the crystal structure of Form I. However, all the H-bonds in the crystal structure of Form II had high propensities of formation, correlating with its greatly increased stability. It is postulated that, had the H-bond propensity data been available during formulation, together with the crystal structure of Form I, then the high possibility of the existence of a second, more stable polymorph would have been recognized and further crystallization experiments carried out. The H-bond propensity methodology is now being added to the Mercury program as an additional computational aid in drug development and formulation.

\section{Future perspective}

Crystal structure data and the knowledge derived from these data continue to make crucial contributions in drug discovery. As our abilities to retrieve and process this information continue to improve, ever more sophisticated questions can be answered efficiently. Crystal structure knowledge, at both the molecular and supramolecular levels, is embedded in procedures that aid rational drug discovery and development on a day-to-day basis. In both areas, the provision of basic structural information will always remain important and the CCDC is embarking on a program of development of the

\section{Bibliography}

Papers of special note have been highlighted as:

- of interest

1 Raymo C. The Virgin and the Mousetrap: Essays in Search of the Soul of Science. Viking Books NY, USA, 154 (1991).

2 White PS, Rodgers JR, Le Page Y. CRYSTMET: a database of the structures and powder patterns of metals and intermetallics. Acta Cryst. B58, 343-348 (2002).
CSD itself. This development will re-engineer the database infrastructure to make database production more automatic, improve its literature currency and bring data to the user more rapidly and in more accessible forms.

In terms of applications research, it is clear that the crystalline nature of many API formulations means that the CSD in particular has much to offer the drug-development scientist. The CCDC has recently established a Crystal Form Consortium, within which industrial development scientists can help to shape the future, particularly in areas of structure prediction and polymorph screening. Over the next few years, the CCDC will be consolidating its predictive capabilities for hydrogen-bonded structures and generalizing these approaches to encompass other interaction types. In drug discovery, the CCDC has an active program for extending the capabilities of its protein-ligand docking software to incorporate improved scoring functions, ensemble docking and methodologies for analyzing the results of high-throughput 'virtual screening', for example.

In both discovery and development, the CSD provides information that increases in breadth and depth as each new structure is added to the collection. Thus, the development of the CSD and its value to pharmaceutical research are synergistic and it is expected that these informatics approaches will progress significantly over the next decade, during which the CSD will expand rapidly to 1 million small-molecule structures.

\section{Financial \& competing interests disclosure}

The authors are currently employees of the Cambridge Crystallographic Data Centre. The authors have no other relevant affiliations or financial involvement with any organization or entity with a financial interest in or financial conflict with the subject matter or materials discussed in the manuscript apart from those disclosed.

No writing assistance was utilized in the production of this manuscript.

Belsky A, Hellenbrandt M, Karen VL, Luksch P. New developments in the Inorganic Crystal Structure Database (ICSD): accessibility in support of materials research and design. Acta Cryst. B58, 364-369 (2002).

4 Berman HM, Olson WK, Beveridge DL et al. The Nucleic Acid Database: a comprehensive relational database of three-dimensional structures of nucleic acids. Biophys. J. 63, 751-759 (1992).

5 Berman HM, Westbrook J, Feng, Z et al. The Protein Data Bank. Nucleic Acids Res. 28, 235-242 (2000). 
6 Allen FH. The Cambridge Structural Database: a quarter of a million crystal structures and rising. Acta Cryst. B58, 380-388 (2002).

7 Bruno IJ, Cole JC, Edgington PR et al. New software for searching the Cambridge Structural Database and visualizing crystal structures. Acta Cryst. B58, 389-397 (2002).

8 Macrae CF, Bruno IJ, Chisholm JA et al. Mercury CSD2.0 - new features for the visualization and investigation of crystal structures. J. Appl. Cryst. 41, 466-470 (2008).

9 Bruno IJ, Cole JC, Kessler, M et al. Retrieval of crystallographically-derived molecular geometry information. J. Chem. Inf. Comput. Sci. 44, 2133-2144 (2004).

- Detailed description of the MOGUL knowledge base of intramolecular geometry.

10 Bruno IJ, Cole JC, Lommerse JPM, Rowland RS, Taylor R, Verdonk ML. IsoStar: a library of information about nonbonded interactions. J. Comput. Aided Mol. Des. 11, 525-537 (1997).

- Detailed description of the IsoStar knowledge base of intermolecular interactions.

11 Thomas IR, Bruno IJ, Cole JC, Macrae CF, Pidcock E, Wood PA. WebCSD: the online portal to the Cambridge Structural Database. J. Appl. Cryst. 43, 362-366 (2010).

12 Brameld A, Kuhn B, Reuter DC, Stahl M. Small molecule conformational preferences derived from crystal structure data: a medicinal chemistry focused analysis. J. Chem. Inf. Model. 48, 1-24 (2008).

- Comprehensive review of conformational applications of the CSD in drug discovery, written from the perspective of a medicinal chemist.

13 Lipinski CA, Lombardo F, Dominy BW, Feeney PJ. Experimental and computational approaches to estimate solubility and permeability in drug discovery and development settings. Adv. Drug Dev Rev. 23, 3-25 (1997).

14 Ghose AK, Viswanadhan VN, Wendoloski JJ. A knowledge-based approach in designing combinatorial or medicinal chemistry libraries for drug discovery. J. Combin. Chem. 1, 55-68 (1999).

15 Allen FH, Galek PTA, Wood PA. Energy matters! Cryst. Rev. (2010) (In Press).

16 Taylor R. Life science applications of the Cambridge Structural Database. Acta Cryst. D58, 879-888 (2002).

17 Klebe G, Meitzner T. A fast and efficient method to generate biologically relevant conformations. J. Comp. Aided Mol. Des. 8, 583-606 (1994).

18 Feuston BP, Miller MD, Culberson JC, Nachbar RB, Kearsley SK. Comparison of knowledge-based and distance geometry approaches for generation of molecular conformations. J. Chem. Inf. Comput. Sci. 41, 754-763 (2001).
19 Wong RW, Allen FH, Willett P. The scientific impact of the Cambridge Structural Database: a citation-based study. J. Appl. Cryst. (2010) (In Press).

20 Allen FH, Taylor R. Research applications of the Cambridge Structural Database. Chem. Soc. Rev. 33, 463-475, 2004.

21 Bissantz C, Kuhn B, Stahl M. A medicinal chemist's guide to molecular interactions. J. Med. Chem. DOI: $10.1021 / j m 100112 \mathrm{j}$ (2010) (Epub ahead of print).

22 Kuhn B, Mohr P, Stahl M. Intramolecular hydrogen bonding in medicinal chemistry. J. Med. Chem. 53, 2601-2611 (2010).

23 Verdonk ML, Cole JC, Taylor R. SuperStar: a knowledge-based approach for identifying interaction sites in proteins. J. Mol. Biol. 289, 1093-1108 (1999).

24 Mohammadi M, McMahon G, Sun, L et al. Structures of the tyrosine kinase domain of fibroblast growth factor receptor in complex with inhibitors. Science 276, 955-960 (1997).

25 Jones G, Willett P, Glen RC, Leach AR, Taylor R. Development and validation of a genetic algorithm for flexible docking. J. Mol. Biol. 267, 727-748 (1997).

- Complete description of the GOLD software for protein-ligand docking.

26 Rarey M, Kramer B, Lengauer T, Klebe G. A fast flexible docking method using an incremental construction algorithm. J. Mol. Biol. 261, 470-489 (1996).

27 Velec HF, Gohlke H, Klebe G. DrugScore (CSD): a knowledge-based scoring function derived from small molecule crystal data with superior recognition rate of near-native ligand poses and better affinity prediction. J. Med. Chem. 48, 6296-6303 (2005).

28 David WIF, Shankland K, van de Streek J, Pidcock E, Motherwell WDS, Cole JC. DASH: a program for crystal structure determination from powder diffraction data. J. Appl. Cryst. 39, 910-915 (2006).

29 Chisholm J, Pidcock E, van de Streek J, Infantes L, Motherwell S, Allen FH. Knowledge-based approaches to crystal design. CrystEngComm. 8, 11-28 (2006).

30 Galek PTA, Allen FH, Fábián L, Feeder N. Knowledge-based H-bond prediction to aid experimental polymorph screening. CrystEngComm. 11, 2634-2639 (2009).

\section{- Website}

101 The Pfizer Institute for Pharmaceutical Materials Science www.msm.cam.ac.uk/pfizer/ 